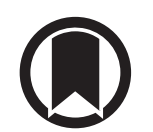

CrossMark

\title{
Maternal nutrition and child respiratory outcomes: paradigms of lung health and disease
}

\author{
Corrine Hanson ${ }^{1}$ and Emily Brigham $\mathbb{B}^{2}$
}

Affiliations: ${ }^{1}$ Division of Medical Nutrition Education, College of Allied Health Professions, University of Nebraska Medical Center, Omaha, NE, USA. ${ }^{2}$ Division of Pulmonary and Critical Care, Johns Hopkins University School of Medicine, Baltimore, MD, USA.

Correspondence: Corrine Hanson University of Nebraska Medical Center, Pulmonary Medicine, 985885 Nebraska Medical Center, Omaha, NE, USA. E-mail: ckhansondunmc.edu

@ERSpublications

A new study shows diet during pregnancy may influence lung health in future generations http://bit.ly/2FEOhlS

Cite this article as: Hanson C, Brigham E. Maternal nutrition and child respiratory outcomes: paradigms of lung health and disease. Eur Respir J 2020; 55: 1902437 [https://doi.org/10.1183/13993003.02437-2019].

Given the large public health burden of obstructive lung diseases, such as asthma and COPD, considerable research has been conducted to identify targeted interventional strategies, with nutrition and dietary intake receiving increasing amounts of attention. Diets rich in antioxidants and omega- 3 fatty acids, and low in saturated fats and processed foods (i.e. the Mediterranean diet) have been proposed as "respiratory healthy" diets, possibly attenuating the inflammatory response and potentially protecting against disease incidence, prevalence, and morbidity [1-5].

Timing of exposures such as diet is an important consideration given multiple plausible windows of susceptibility. Because pulmonary structure and early function is established in utero, and structural abnormalities may confer the presence of, or vulnerability to, disease, maternal diet during pregnancy and thereby fetal exposure is a time of potential high impact. Respiratory symptoms often manifest in the early months of life, suggesting that the processes leading to their development may be influenced by prenatal exposures [6], and maternal diet is a potentially modifiable prenatal exposure that has been shown to influence birth outcomes $[7,8]$. Prenatal nutrient and food intake has been linked to outcomes of allergic disease, wheeze and asthma [9-11]; investigations of prenatal diet patterns, including a Mediterraneanstyle diet, are limited and provide mixed evidence for an association with childhood wheeze [12, 13]. Further evidence supports the role of diet as a modifying factor in those with prevalent disease; observational work demonstrates the positive and negative effects of foods, nutrients, and patterns typical of "healthy" and "unhealthy" intake, respectively, on asthma and COPD respiratory morbidity [14-18].

Much less is known about the effect of nutrition on optimisation of lung health apart from defined disease pathology, including at what point in the life course nutritional factors may be most influential. The "life course" conceptual model, examining the long-term effects of diverse exposures across the life span, is appropriate to consider [19]. Pregnancy and early life may represent a critical period for lung health in the life course approach, when an exposure acting during this time has the potential for durable effects on lung health. For example, small improvements in the lung function of newborns may theoretically translate into attainment of full potential for lung growth, providing a greater ability to withstand the effects of 
deleterious exposures encountered over the lifespan and reducing the likelihood of later crossing the threshold for disease diagnosis. Given the large number of individuals affected by lung disease, even incremental shifts at the centre of a bell-shaped curve can have important consequences, to the benefit or detriment of population health.

Submaximal peak lung health achieved early in life and loss of lung function over time are recognised risk factors for the development of disease in adolescence and adulthood. Using the Coronary Artery Risk Development in Young Adults (CARDIA) cohort study, JACOBs et al. [20] examined the associations between peak lung health, loss of lung health, and a variety of clinical outcomes. The decline from peak forced vital capacity (FVC) attained in young adulthood was associated with future hypertension, while declines in FVC in young adulthood have also been associated with hypertrophic, high-output cardiac phenotype with increased left ventricular mass and signs of diastolic dysfunction [21]. These documented adverse outcomes associated with reduced lung function, even when within a "normal" range, provide rationale that further consideration should be given to the context of "lung health" rather than "lung disease".

In this issue of the European Respiratory Journal, BÉDARD et al. [22] use a well-characterised birth cohort (the Avon Longitudinal Study of Parents and Children, ALSPC) to examine the relationship between a Mediterranean diet during pregnancy and disease outcomes, as well as lung function at 8 years of age in offspring. While there were no associations between a Mediterranean diet and asthma or other allergic outcomes, the authors do report the important finding that a Mediterranean diet during pregnancy was associated with better lung function in childhood, as measured by maximal mid-expiratory flow $\left(\mathrm{FEF}_{25-75}\right)$. This places these findings squarely within the context of "lung health", and identifies potential interventions that could serve to maximise lung function early in life, even in the absence of any disease. These findings complement results of a recent study designed to determine the impact of a more pro-inflammatory diet during pregnancy. Within Project Viva, a longitudinal cohort of mother and child pairs, maternal dietary intake during pregnancy was used to calculate individual Dietary Inflammatory Index scores, representing the inflammatory potential of diet. Data from 1424 mothers was compared to respiratory outcomes in both early and mid-childhood. The authors found that a pro-inflammatory diet in the first trimester of pregnancy was associated with lower measures of $\mathrm{FEF}_{25-75}$ in the offspring at an average of 7 years of age [23]. Notably, $\mathrm{FEF}_{25-75}$ values, representing small airway patency and significantly linked with dietary exposures in both studies, are predictive of asthma severity and control in paediatric populations with a normal forced expiratory volume in $1 \mathrm{~s}\left(\mathrm{FEV}_{1}\right)$ [24] but are not currently incorporated into guidelines for clinical care.

While the types of exposures between these two studies is different (a diet pattern versus an index of the inflammatory potential of diet), both add to the body of evidence that support a "total diet" concept, recognising that nutrients occur simultaneously with many other compounds in foods. It is possible that the effects associated with a certain nutrient are, in fact, a result of another compound or group of compounds; or the effects of multiple compounds could be additive, synergistic, or interact in other ways. Whereas individual nutrient supplementation trials have largely failed to demonstrate benefit for lung health, several small trials of whole food and diet pattern intervention have shown promise. In Australia, 137 adults with asthma were randomised to either $\leqslant 1$ serving fruit and 2 servings vegetables daily (low antioxidant) versus 2 servings fruit and 5 servings vegetables daily (high antioxidant). Percent predicted $\mathrm{FEV}_{1}$ was significantly reduced in the low compared to the high antioxidant group [25]. Small pilot trials of healthy diets in US populations demonstrate encouraging trends toward improvements in lung function and asthma control $[26,27]$.

The authors also hypothesised that a higher Mediterranean diet score during pregnancy might be particularly beneficial when a fetus was co-exposed to cigarette smoke. However, they found the opposite to be true: an association was only seen among mothers who had not smoked during pregnancy. Smokers will carry not only a burden of general oxidants, but also a burden of oxidants generated from cigarette smoke directly, and from the inflammation that smoking induces. Smokers have also been shown to be have lower serum levels of nutritional antioxidants than their non-smoking counterparts, potentially inhibiting their antioxidant capacity. Studies have reported that smokers have lower plasma carotenoid concentrations compared to non-smokers, with a higher numbers of cigarettes per day leading to a corresponding decrease in serum carotenoids in a dose-dependent manner [28]. The highly oxidative nature of tobacco smoke has been shown to destroy $\beta$-carotene and other carotenoids in human plasma in vitro [29], which may contribute to a reduction in serum levels. The association of serum $\beta$ - carotene and $\mathrm{FEV}_{1}$ has been shown to be weaker in smokers when compared to non-smokers, and the strength of the relationship decreased as the amount of smoking increased [30]. It is possible that the children of smokers in this study did not benefit from a Mediterranean diet if the overall oxidative burden was such that it overwhelmed any potential benefits from diet, or that heterogeneity of smoke exposure (either amount or timing) in the smoking group significantly confounded associations. Regardless, the results suggest that 
smoking and perhaps other airborne pollutant exposures may be an important consideration in the evaluation of nutritional effects on respiratory outcomes.

Prior publications [23, 31] and the new results by BÉDARD et al. [22] in this issue of the European Respiratory Journal suggest that maternal diet during pregnancy can influence the lung function of future generations. While further longitudinal studies will help to clarify the nature of these associations, well-designed randomised controlled trials will be needed to ascertain causality of the association observed in the epidemiological literature. In the meantime, it seems prudent to recommend balanced diets rich in antioxidant and anti-inflammatory nutrients during pregnancy.

Conflict of interest: None declared.

\section{References}

1 Varraso R, Fung TT, Barr RG, et al. Prospective study of dietary patterns and chronic obstructive pulmonary disease among US women. Am J Clin Nutr 2007; 86: 488-495.

2 Shaheen SO, Jameson KA, Syddall HE, et al. The relationship of dietary patterns with adult lung function and COPD. Eur Respir J 2010; 36: 277-284.

3 Papamichael MM, Itsiopoulos $\mathrm{C}$, Susanto $\mathrm{NH}$, et al. Does adherence to the Mediterranean dietary pattern reduce asthma symptoms in children? A systematic review of observations studies. Public Health Nutr 2017; 20: 2722-2734.

4 Lemoine C, Brigham E, Woo H, et al. Relationship between omega-3 and omega- 6 fatty acid intake and COPD morbidity. Ann Am Thorac Soc 2019; in press [https://doi.org/10.1513/AnnalsATS.201910-740RL].

5 Brigham EP, Steffen LM, London SJ, et al. Diet pattern and respiratory morbidity in the Atherosclerosis Risk in Communities study. Ann Am Thorac Soc 2018; 15: 675-682.

6 Romieu I, Torrent M, Garcia-Esteban R, et al. Maternal fish intake during pregnancy and atopy and asthma in infancy. Clin Exp Allergy 2007; 37: 518-525.

7 Bedard A, Northstone K, Holloway J, et al. Maternal dietary antioxidant intake in pregnancy and childhood respiratory and atopic outcomes: birth cohort study. Eur Respr J 2018; 52: 1800507.

8 Sen S, Rifas-Shiman SL, Shivappa N, et al. Associations of prenatal and early life dietary inflammatory potential with childhood adiposity and cardiometabolic risk in project viva. Pediatr Obes 2018; 13: 292-300.

9 Maslova E, Rifas-Shiman SL, Oken E, et al. Fatty acids in pregnancy and risk of allergic sensitization and respiratory outcomes in childhood. Ann Allergy Asthma Immunol 2019; 122: 120-122. e3.

10 Sordillo JE, Switkowski KM, Coull BA, et al. Relation of prenatal air pollutant and nutritional exposures with biomarkers of allergic disease in adolescence. Sci Rep 2018; 8: 10578.

11 Bunyavanich S, Rifas-Shiman SL, Platts-Mills TA, et al. Peanut, milk, and wheat intake during pregnancy is associated with reduced allergy and asthma in children. J Allergy Clin Immunol 2014; 133: 1373-1382.

12 Chatzi L, Garcia R, Roumeliotaki T, et al. Mediterranean diet adherence during pregnancy and risk of wheeze and eczema in the first year of life: INMA (Spain) and RHEA (Greece) mother-child cohort studies. Br J Nutr 2013; 110: 2058-2068.

13 Lange NE, Rifas-Shiman SL, Camargo CA, et al. Maternal dietary pattern during pregnancy is not associated with recurrent wheeze in children. J Allergy Clin Immunol 2010; 126: 250-255. 255.e1.

14 Yazdanpanah L, Paknahad Z, Moosavi AJ, et al. The relationship between different diet quality indices and severity of airflow obstruction among COPD patients. Med J Islam Repub Iran 2015; 30: 380.

15 Brigham EP, Woo H, McCormack M, et al. Omega-3 and omega-6 intake modifies asthma severity and response to indoor air pollution in children. Am J Respir Crit Care Med 2019; 199: 1478-1486.

16 Barros R, Moreira A, Fonseca J, et al. Adherence to the Mediterranean diet and fresh fruit intake are associated with improved asthma control. Allergy 2008; 63: 917-923.

17 Bilbeisi AHH E, Albelbeisi A, Hosseini S, et al. Dietary pattern and their association with level of asthma control among patients with asthma and Al-Shifa Medical Complex in Gaza Strip, Palestine. Nutr Metab Insights 2019; 12: 1178638819841394.

18 Varraso R, Kauffmann F, Leynaert B, et al. Dietary patterns and asthma in the E3N study. Eur Respir J 2009; 33: 33-41.

19 Camargo C, Budinger S, Escobar G, et al. Promotion of lung health: NHLBI Workshop on the Primary Prevention of Chronic Lung Diseases. Ann Am Thorac Soc 2014; 11: Suppl. 3, S125-S128.

20 Jacobs DR Jr, Yatsuya H, Hearst MO, et al. Rate of decline of forced vital capacity predicts future arterial hypertension: the Coronary Artery Risk Development in Young Adults Study. Hypertension 2012; 59: 219-225.

21 Cuttica MJ, Colangelo LA, Shah SJ, et al. Loss of lung health from young adulthood and cardiac phenotypes in middle age. Am J Respir Crit Care Med 2015; 192: 76-85.

22 Bédard A, Northstone K, Henderson AJ, et al. Mediterranean diet during pregnancy and childhood respiratory and atopic outcomes: birth cohort study. Eur Respir J 2020; 55: 1901215.

23 Hanson C, Rifas-Shiman S, Shivappa N, et al. Associations of prenatal dietary inflammatory potential with wheeze trajectory in Project Viva. J Allergy Clin Immun in Practice 2019; in press [https://doi.org/10.1016/j.jaip.2019.10.010].

24 Rao DR, Gaffin JM, Baxi SN, et al. The utility of forced expiratory flow between $25 \%$ and $75 \%$ of vital capacity in predicting childhood asthma morbidity and severity. J Asthma 2012; 49: 586-592.

25 Wood LG, Garg ML, Smart JM, et al. Manipulating antioxidant intake in asthma: a randomized controlled trial. Am J Clin Nutr 2012; 96: 534-543.

26 Brigham EP, Matsui EC, Appel LJ, et al. A pilot feeding study for adults with asthma: the Health Eating Better Breathing Trial. PLoS One 2017; 12: e180068.

27 Ma J, Strub P, Lv N, et al. Pilot randomized trial of a healthy eating behavioral intervention in uncontrolled asthma. Eur Respir J 2016; 47: 122-132.

28 Fukao A, Tsubono Y, Kawamura $\mathrm{M}$, et al. The independent association of smoking and drinking with serum beta-carotene levels among males in Miyagi, Japan. Int J Epidemiol 1996; 25: 300-306. 
29 Baker DL, Krol ES, Jacobsen N, et al. Reactions of beta-carotene with cigarette smoke oxidants: identification of carotenoid oxidation products and evaluation of the prooxidant/antioxidant effect. Chem Res Toxicol 1999; 12: 535-543.

$30 \mathrm{Hu} \mathrm{G}$, Cassano PA. Antioxidant nutrients and pulmonary function: the third national health and nutrition examination survey (NHANES III). Am J Epidemiol 2000; 151: 975-981.

31 Castro-Rodriguez JA, Garcia-Marcos L, Alfonseda Rojas JD, et al. Mediterranean diet as a protective factor for wheezing in preschool children. J Pediatr 2008; 152: 823-828. 828.e1-2. 